\title{
ESTRATEGIA PARTICIPATIVA PARA ESTUDIANTES DE LA CARRERA DE ESTUDIOS SOCIOCULTURALES
}

\section{STRATEGY FOR PARTICIPATION OF THE STUDENT OF SOCIOCULTURAL STUDIES CARRIER}

\author{
Gretel Báez Padrón*
}

RESUMEN

El presente artículo aborda la necesidad de insertar a los estudiantes de la carrera de Estudios Socioculturales del Centro Universitario Municipal (cum) de San Cristóbal, en las instituciones socioculturales existentes en el Municipio, a través de tres estrategias específicas: la primera dirigida al acercamiento a las instituciones; la segunda dirigida a la capacitación de estudiantes y profesores y la tercera dirigida a la gestión de proyectos culturales, donde los propios estudiantes sean protagonistas activos de estos, los cuales estarán dirigidos a lograr un vínculo más estrecho entre el estudiante, la institución y la comunidad.

PALABRAS CLAVE: CUBA * ESTUDIANTE * UNIVERSIDAD * GESTIÓN CULTURAL * PARTICIPACIÓN CULTURAL * ESTRATEGIA DE ENSEÑANZA

\section{ABSTRACT}

This article studies the necessity of introduce to the students of Sociocultural Studies carrier of the University of San Cristobal, in the sociocultural institutions of the town, through three specific strategies: first the union between the students and the institution; second, training of students and teachers and third, management of socio-cultural projects, in which the students are protagonist.

KEYWORDS: CUBA * STUDENT * UNIVERSITY $*$ CULTURAL MANAGEMENT $*$ CULTURAL PARTICIPATION $*$ TEACHING STRATEGY

\footnotetext{
* Centro Universitario Municipal (cum) de San Cristóbal, Cuba.
} gretelb@isch.edu.cu 


\section{INTRODUCCIÓN}

La problemática referida a la participación estudiantil ha resultado un tema polémico en los últimos años, por lo que el siguiente artículo pretende diseñar una estrategia para incrementar la participación de los estudiantes de la Carrera de Estudios Socioculturales del Centro Universitario Municipal de San Cristóbal (Cuba), en la gestión de las instituciones socioculturales existentes en el Municipio, teniendo en cuenta que el desarrollo de estas instituciones debe sustentarse en la participación protagónica del estudiante de la carrera de Estudios Socioculturales, así como, en la organización, proyección y dinamización de las actividades que se realizarán. Además, el diseño de una estrategia permitirá la inserción de estos estudiantes en la gestión de estas instituciones a través de tres estrategias específicas, que permitan contribuir a su formación e identificación con el proceso de gestión de las instituciones, en función de elevar el desarrollo sociocultural de las mismas, el desarrollo sociocultural universitario y el desarrollo local.

El perfil de esta titulación parte del objeto de trabajo de este profesional, que implica realizar trabajo sociocultural comunitario, el cual implica realizar trabajo social comunitario, investigación social, actividades de promoción, animación y gestión cultural, donde su modo de actuación es precisamente, la realización de un trabajo especializado de detección, investigación e intervención sociocultural. Esto es lo que debiera ser, pero estos estudiantes por ser estudiantes de la universalización, su plan de estudios es diferente y no existe en este la disciplina principal integradora, la cual que vincula la teoría y la práctica insertándolos, en las instituciones socioculturales para realizar el trabajo sociocultural. De ahí la necesidad de vincular a estos estudiantes a las instituciones socioculturales existentes en el Municipio y lograr que sean protagonistas en sus actividades.

\section{MARCO TEÓRICO}

Como antecedente de esta investigación se encuentra el Programa Nacional de
Extensión Universitaria (2004), el cual aborda el concepto de instituciones socioculturales.

En cuanto al tema de la participación, se han referido diversos autores, como Fernando de la Riva (1994), Giuletta Fadda (1990) y Cecilia Linares (1996), quienes han desarrollado diferentes investigaciones y ponencias.

Desde una perspectiva más amplia, la participación debe considerarse como una necesidad social en función de transformar el medio. El hombre como ser social que necesita relacionarse, compartir con los demás y por ser ciudadano libre, tiene derecho a estar informado, operar e intervenir en la vida política, social y cultural de la comunidad. Participar implica un mejor conocimiento de la realidad y contribuye a la educación cívica de los ciudadanos. Además, es un medio para lograr el reparto equitativo de los beneficios $y$ una forma de tomar parte en el proceso de toma de decisiones.

Lo expuesto anteriormente corrobora el concepto sobre la participación de Linares, para quien este término debe caracterizarse por la masividad $y$ la diversidad con la finalidad de implicar a la mayor cantidad de personas en actividades donde puedan exponer sus opiniones e intereses dentro de los niveles organizativos alcanzados. Considera que participar es:

El derecho de opinar, decidir sobre los objetivos, metas, planes $y$ acciones en cada una de las etapas del proceso, así como la posibilidad de intervenir en la toma de decisiones en las diferentes fases de la búsqueda de soluciones en el trazado de una estrategia, en la ejecución de un programa y sus beneficios (2004).

Teniendo en cuenta lo anterior, participar es algo más que asistir o estar presentes, es tomar parte, intervenir, implicarse, colaborar, decidir, gestionar y accionar, la presencia debe ser activa comprometiendo a la persona en mayor o menor medida.

Según de la Riva (1994), para que la participación se produzca es necesario que los miembros a quienes se dirige la acción quieran tomar parte, intervenir en la acción participativa. La motivación constituye una condición necesaria para que se produzca la participación, 
este autor la considera como: "el conjunto de impulsos, necesidades, deseos, razones, intereses, motivos, capaces de movilizar a la acción, en este caso, la participación".

Según este mismo autor, la motivación se conforma en torno a tres ejes fundamentales: el interés subjetivo o ideológico, el cual se da cuando la propuesta participativa coincide con los ideales o ideas de las personas a quien va dirigida la acción; la satisfacción socio afectiva, que se da cuando la propuesta permite o facilita el reconocimiento social y la percepción de rentabilidad, la cual se da cuando la propuesta es creíble, cuando se piensa que es útil.

Según González y González, la participación es "la intervención activa de los diferentes actores sociales en los procesos dinámicos de construcción y transformación de la sociedad. Es un compromiso para la acción, una actitud para asumir la realidad, compatibilizar intereses e involucrarse en el cambio" (2007: 8).

Este concepto asume la participación, en cuanto a la intervención activa de los actores sociales en los procesos de transformación de la realidad.

Promover la participación supone que existan posibilidades reales de participar, así como la habilidad que se desarrolla, se perfecciona y se facilita a través de tres procesos básicos: el conocimiento de la realidad, el flujo de comunicación eficiente y la educación para la participación.

Además, es importante conocer que la participación juega un papel importante en el desarrollo de los proyectos sociales, en tanto se logre el protagonismo de los estudiantes en función de la transformación del medio. La participación potencia la capacidad de diálogo, evaluación, planificación y trabajo en común.

En el caso en estudio, la Licenciatura en Estudios Socioculturales supone la formación científica y ética de los futuros graduados que ante todo, deben tener conciencia $y$ actuar en la práctica social, según dos principios básicos: el protagonismo real de las personas, grupos y comunidades, así como, la participación activa de todos en los procesos socioculturales.

Esta carrera surge en el año 1999, en la provincia de Cienfuegos y un año más tarde, se abre en las universidades que solamente impartían las ciencias técnicas, con el objetivo de humanizar o socializar la cultura general de dichos centros y graduar profesionales más integrales.

Aunque el surgimiento de la carrera ha constituido un cambio cualitativo y cuantitativo, aún es insuficiente la participación de los estudiantes de dicha carrera en la gestión de las instituciones socioculturales existentes en el Municipio.

Por lo tanto, se planteó el siguiente problema de investigación: ¿cómo lograr la participación de los estudiantes de la carrera de Estudios Socioculturales del Centro Universitario Municipal de San Cristóbal, en la gestión de las instituciones socioculturales existentes en el Municipio? El objetivo que se ha trazado es diseñar una estrategia que permita la participación de los estudiantes en la gestión de las instituciones socioculturales.

\section{IDEAS A DEFENDER}

$\diamond \quad$ El desarrollo de las instituciones socioculturales existentes en el Municipio como una dimensión de la extensión universitaria, debe sustentarse en la participación del estudiante de la carrera de Estudios Socioculturales, en la organización, la proyección y la dinamización de las actividades realizadas.

$\diamond \quad$ El diseño de una estrategia permitirá la inserción de los estudiantes de la carrera de Estudios Socioculturales en la gestión de las instituciones socioculturales, a través de tres estrategias específicas, las cuales permitan contribuir a su formación e identificación con el proceso de gestión de las instituciones, con el fin de elevar el desarrollo sociocultural de las mismas y el desarrollo sociocultural universitario.

La carrera de Estudios Socioculturales no tiene antecedentes directos en el sistema de educación superior cubano; sin embargo, es integradora de aspectos principales del sistema de conocimientos, habilidades y modos de actuación de las licenciaturas en Letras, Historia del Arte, Historia, Sociología y Ciencias 
Sociales, al mismo tiempo, complementa la formación de profesionales del sistema de cultura $y$ turismo que no poseen este perfil en el tercer nivel de enseñanza.

El objeto de trabajo de este profesional es el trabajo sociocultural comunitario. Su modo de actuación es la realización de un trabajo especializado de detección, investigación e intervención sociocultural; por ello, el objeto de la profesión es precisamente la intervención social comunitaria que implica el trabajo con grupos étnicos, género, territorios, generaciones, lo urbano y lo rural, el turismo, etc.

Esta carrera prepara profesionales capaces de realizar trabajo social comunitario, asesorías, investigación social, promoción, animación, gestión cultural y turística e incluso labores de formación docente y de extensión en las esferas social, cultural y turística.

Todos estos elementos antes mencionados son tomados del Plan de Estudios de la titulación Lic. en Estudios Socioculturales, que es un documento oficial y estatal, por lo que debe ser referenciado y explicitado las modificaciones realizadas por el Ministerio de Educación Superior en Cuba.

Para realizar el trabajo de intervención sociocultural, se requiere del dominio de los siguientes campos de acción: cultura, historia, teoría y metodología sociocultural.

Los campos de acción abarcan todos los ámbitos sociales, muy especialmente, las instituciones culturales y educativas, los centros de trabajo y los consejos populares. Estos espacios de alta concentración de personas y grupos, cualitativamente significativos en el desarrollo sociocultural, deben ser priorizados para contribuir al proceso acelerado de socialización de la cultura. Son facilitadores de la afirmación y construcción de la identidad personal y social, la apreciación y creación de valores éticos y estéticos, así como, el despliegue de la creatividad, la comunicación y la participación social, formaciones todas de amplia aplicación en el porvenir de la sociedad cubana.

Deben ser capaces de integrar grupos de trabajo interdisciplinarios en los campos y esferas de actuación determinadas, así como realizar diagnósticos de problemas con fines de intervención-transformación.
Sus esferas de actuación son aquellas vinculadas tanto a la concepción cultural del individuo, como a las zonas de relación sociocultural, las cuales demandan de un trabajo especializado de detección, investigación e intervención, tales como los ámbitos: sociocultural, el político ideológico, el turismo y la educación. De ahí la necesidad de que el estudiante de esta carrera durante su vida estudiantil se inserte en las instituciones socioculturales existentes en el municipio, con el fin de familiarizarse con este trabajo y lograr un dominio pleno de su futura actuación profesional.

Las instituciones socioculturales existentes en el municipio de San Cristóbal constituyen un espacio físico capaz de elevar la cultura universitaria, siempre que sean utilizadas con este fin $y$ no se limiten a cumplir con sus funciones administrativas, dejando a un lado la realización de actividades culturales, como es el caso del museo, la casa de cultura y la librería, los cuales dejan de lado la realización de actividades de promoción y animación sociocultural que involucran al público y fundamentalmente, a los estudiantes de la carrera de Estudios Socioculturales. Las mismas están encargadas de incrementar la cultura en la comunidad y a la vez, retroalimentarse de los estudiantes para mejorar su desempeño y desarrollo.

Una vía efectiva para lograr un vínculo más estrecho entre las instituciones y el estudiante, pudiera ser la realización de proyectos que involucren a las instituciones, como los proyectos sociales $y$ otros que se desarrollen por interés propio. Estos proyectos pueden llevarse a cabo teniendo como escenario dichas instituciones, con el fin de lograr el desarrollo sociocultural de ambos, contribuyendo así al desarrollo sociocultural universitario y al desarrollo local.

El desarrollo sociocultural es visto por Russeau (1999) como:

El conjunto de ideas sistematizadas que intentan dar explicación a los procesos de evolución y/o transformación, espontánea o inducida, de una cultura determinada, así como también a los factores conformantes de dicho proceso. 
También es entendido como una meta o un fin último a alcanzar por una sociedad (1999: 48).

Se considera que el mismo debe estar orientado hacia la transformación del sector, de la dimensión, de las áreas y los procesos socioculturales, además de ser un proceso de despliegue de las potencialidades de creación $y$ expresión de una sociedad, esencialmente diversas y multifacéticas, forjadas en su propia historia $y$ transformadas permanentemente en el acontecer de su vida sociocultural. Al mismo tiempo es la acción de los diferentes grupos sociales para transformar el sentido y los estilos de sus vidas.

Según Ballester, el desarrollo sociocultural se considera como:

Crecimiento de las potencialidades propias de cada cultura... debe lograrse a través de diferentes funciones de la educación superior donde la extensión universitaria está considerada como parte del sistema de interacciones entre la UniversidadSociedad, con un carácter integrador de la función académica e investigativa que contribuye significativamente a viabilizar la preservación y promoción de la cultura, tanto en el centro docente como en su entorno social (1996: 1).

La cultura en el contexto del desarrollo constituye un factor esencial; es un proceso global, vinculado a los propios valores de cada sociedad y exige una participación activa de los grupos e individuos, quienes son tanto los autores como los beneficiarios de ese proceso.

Se debe considerar que todo desarrollo implica cambios profundos en las estructuras socioculturales y económicas que serán el resultado de fuerzas internas de la sociedad (aunque también pueden ser estimuladas por fuerzas externas).

El desarrollo sociocultural es un proceso dirigido a:

$\diamond \quad$ Potenciar las capacidades creadoras, la circulación y la utilización de los valores culturales. $\diamond \quad$ Rescatar, preservar y revitalizar el patrimonio cultural.

$\diamond \quad$ Desarrollar la participación social.

$\diamond \quad$ Potenciar el desarrollo de las restantes esferas de la vida.

$\diamond \quad$ Integrar diferentes fuerzas sociales.

Según el Programa Nacional de Extensión Universitaria:

...las Instituciones Socioculturales se identifican en dos vertientes: las que constituyen locaciones que ofrecen una programación cultural y deportiva (teatro, museo, bibliotecas, sala de historia, galerías, plazas, memoriales, áreas deportivas, etc.) y las asociadas a colectivos que promueven o ejecutan una expresión o manifestación cultural, deportiva o científica (grupos de teatro, clubes de arte, cátedras honoríficas, coral, talleres de apreciación, equipos deportivos, etc.) $(2004)^{1}$.

Para Marcovitch (2004), la gestión de procesos culturales desde las instituciones debe considerar diferentes aspectos:

$\diamond \quad$ La generación de proyectos, es decir, la elaboración y negociación de propuestas identificando necesidades que se convier$\tan$ en respuestas directas.

$\diamond \quad$ La planificación y administración de los proyectos mediante su integración al proceso de planificación de la organización como un todo, a partir de la elaboración de planes de ejecución.

$\diamond \quad$ El ejercicio de la función de control y seguimiento sobre los mismos, integrándola al sistema de informaciones, administrativas del conjunto de la entidad.

$\diamond \quad$ El establecimiento de una estructura organizacional para la administración de proyectos, mediante la definición de responsabilidades $y$ niveles de autoridad entre los diversos miembros de la organización.

1 Este concepto se asumirá en el transcurso de la investigación, por ser el más integrador 
$\diamond \quad$ Establecimiento de los aspectos administrativos para la ejecución de proyectos, a partir de los elementos más pertinentes en la etapa de ejecución: liderazgo, motivación, conflictos y solución de problemas.

$\diamond \quad$ La determinación de cómo se van a analizar los proyectos, cuándo terminan y cómo se va a efectuar la transferencia de sus resultados.

La gestión es más exitosa, en la medida en que sea mayor la participación de todos los involucrados. Sus dos variables más importantes son los objetivos de la organización y las necesidades que deben ser satisfechas.

Partiendo del concepto expuesto por Fernando de la Riva, se considera que la participación protagónica del estudiante de la carrera de Licenciatura en Estudios Socioculturales en la gestión de las instituciones socioculturales, consiste en ejercer el derecho de opinar y decidir sobre los objetivos, metas y planes de acción que se estructuren para la búsqueda de soluciones que viabilicen la inserción de estas instituciones en el trabajo sociocultural.

Solo cuando la persona percibe claramente que organizarse será útil para sus propios intereses $y$ que la propuesta tiene posibilidades reales de satisfacción a sus necesidades o intereses, su motivación para tomar parte se hará evidente.

Además, es importante contar con los conocimientos, capacidades, habilidades o destrezas necesarias para tomar parte, para intervenir en la propuesta participativa y en la satisfacción de sus motivaciones. Esto conlleva a la formación como segunda condición necesaria para que se produzca la participación.

Aunado a lo anterior, es necesario que existan las ocasiones, los cauces y las estructuras que hagan posible la participación efectiva.

El municipio de San Cristóbal cuenta con una serie de instituciones socioculturales generadoras y promotoras del desarrollo cultural, las cuales deberían estar en función de desarrollar una intensa vida cultural mediante acciones.
La reanimación de las instituciones socioculturales existentes en el municipio de San Cristóbal, mediante la inserción de los estudiantes de la carrera de Estudios Socioculturales en su gestión y junto a las actividades extracurriculares, constituye una vía efectiva en el desarrollo cultural integral de la comunidad universitaria y de la sociedad.

\section{MATERIALES Y MÉTODOS}

Para el desarrollo de la investigación se utilizó el estudio de varios métodos, entre los cuales se encuentra el método Histórico Lógico, el cual permitió analizar la evolución de la participación estudiantil, en este caso en Cuba, así como, la perspectiva actual en que se desenvuelve. Además, permitió conocer la historia de las instituciones socioculturales $y$ su papel en la Universidad. Asimismo, se utilizó el método sistémico estructural para la determinación de la estrategia.

\section{MÉTODOS EMPÍRICOS}

$\diamond \quad$ Entrevistas estructuradas a profesores $y$ directivos de la carrera de Estudios Socioculturales, directivos y trabajadores de las instituciones socioculturales para la constatación del problema y conocer la implicación de los estudiantes de esta carrera en la gestión de las instituciones del Municipio.

$\diamond \quad$ La observación participante para valorar la participación protagónica de los estudiantes de la carrera de Estudios Socioculturales en actividades desarrolladas en las instituciones socioculturales.

$\diamond \quad$ Análisis para la valoración de los documentos sobre el tema de la extensión y el protagonismo estudiantil, además de otras publicaciones de interés.

\section{TÉCNICAS}

La entrevista grupal realizada a estudiantes de la carrera de Estudios Socioculturales para conocer si se vinculan a las instituciones socioculturales existentes en el territorio. Además, permitió combinar elementos de la 
metodología cualitativa para constatar la información aportada.

\section{RESULTADOS Y DISCUSIÓN}

La entrevista grupal se aplicó en el Centro Universitario Municipal de San Cristóbal, a cinco grupos de seis estudiantes cada uno. El año lectivo que se tomó como muestra fue el 4to. año de la carrera, lo que da un total de 30 estudiantes de 4to. año. La mayoría de los estudiantes (22) expresaron que conocían las instituciones, pero se implicaban de forma muy limitada, solo mencionaron la librería, la biblioteca y el museo. Además, manifestaron que han participado en exposiciones de arte $y$ lanzamientos de libros y revistas, los cuales se realizan en la librería esporádicamente.

Opinan que no tienen la preparación suficiente para involucrarse en el proceso de gestión de las instituciones socioculturales, ni tampoco poseen espacios para actuar, siendo ellos los organizadores de las actividades culturales, ya que los directivos de las instituciones no dan espacios para insertarse y en muchas ocasiones, no realizan actividades culturales en las instituciones.

ENTREVISTA REALIZADA A PROFESORES DE LA CARRERA DE ESTUDIOS SOCIOCULTURALES DEL CENTRO UNIVERSITARIO MUNICIPAL DE SAN CRISTÓBAL

Se entrevistaron un total de 10 profesores de la carrera. En estas entrevistas, la mayoría de los profesores (8) coinciden en que los estudiantes están muy alejados de las estas y se involucran muy poco en su gestión. Esto debido a que no cuentan con la preparación requerida para ello, pues no han recibido cursos de promoción ni de gestión, ya que el currículo de la carrera no incluye estas asignaturas $y$ no cuentan con las iniciativas necesarias para llevar a cabo esta labor, por lo que es necesario orientarlos en este sentido.

Asimismo, expresan que existen proyectos de desarrollo sociocultural que tributan al avance de la Universidad como la estrategia de desarrollo de la Universidad y los proyectos educativos de cada brigada. Aunque consideran que pueden hacerse más proyectos en función de apoyar al desarrollo sociocultural universitario.

Todos estuvieron de acuerdo en que la carrera presenta un alto grado de responsabilidad con el desarrollo sociocultural de la Universidad, ya que precisamente surge para promover y elevar su desarrollo. No obstante, se hizo referencia a que se deben hacer mayores esfuerzos para apoyar el desarrollo sociocultural de la Universidad, participar más en el orden artístico literario y promover otras actividades.

\section{ENTREVISTA REALIZADA A DIRECTIVOS DE LA CARRERA DE ESTUDIOS SOCIOCULTURALES DEL CENTRO UNIVERSITARIO MUNICIPAL DE SAN CRISTÓBAL}

Se entrevistaron un total de 6 directivos, los cuales están de acuerdo en que los estudiantes deben ser participantes activos en la vida cultural de las instituciones socioculturales, siendo organizadores $y$ promotores de las actividades que se desarrollen en estas.

\section{ENTREVISTA REALIZADA A TRABAJADORES DE LAS DIFERENTES INSTITUCIONES DEL MUNICIPIO}

Se entrevistaron un total de 10 trabajadores de las siguientes instituciones:
1) Biblioteca
2) Librería
3) Museo
4) Galería de arte
5) Casa de cultura

En el caso de las entrevistas en la biblioteca, se considera que es una institución que juega un papel primordial en el desarrollo sociocultural de la Universidad, no solo por la función instructiva que desarrollan, como el préstamo de libros, búsqueda en Internet y otros servicios, sino también, por las actividades socioculturales que se desarrollan como son: promoción de la lectura, en la cual se trae un libro y se conversa sobre este, se hace un debate sobre el tema fundamental que aborda y sobre su autor; tertulias literarias, tardes literarias, la hora del cuento y el círculo Conservando para el futuro. 
Por su parte, la librería también juega un papel importante, debido a la venta de libros, los cuales proporcionan a los estudiantes el poder ampliar su acervo cultural. Asimismo, las actividades que se realizan como exposiciones de arte, presentaciones de libros y conversatorios que se desarrollan con poetas san cristobalenses.

El personal vinculado al museo y a los diferentes parques, plazas y monumentos, tiene mucha responsabilidad con el desarrollo sociocultural de la localidad $y$ de la Universidad, ya que debe difundir los valores históricos de estas instituciones $y$ realizar actividades con los estudiantes de la carrera de Estudios Socioculturales, donde sean partícipes como promotores y organizadores.

En cuanto a la vinculación de los estudiantes de la carrera de Estudios Socioculturales con las instituciones, se involucran muy poco, consideran que deben insertarse más en todas las instituciones y no solo asistir a las mismas cuando se les orienta un trabajo extraclase o para estudiar, como en el caso de la biblioteca, sino ser promotores, organizadores $y$ espectadores activos de las actividades que en estas se realizan.

Consideran que es importante la participación de estos estudiantes en la gestión de todas las instituciones, ya que contribuirían a elevar su desarrollo sociocultural y ayudarían a que sus actividades presenten mayor calidad, además de promoverlas y organizarlas.

Debido a la insuficiente participación protagónica de los estudiantes de esta carrera en la gestión de las instituciones socioculturales, se hace necesario diseñar una estrategia en la cual se desarrollen acciones encaminadas a insertar a los estudiantes en estas, con el objetivo de reanimarlas y contribuir a su mejor desempeño, así como al desarrollo sociocultural universitario $y$ al desarrollo local de la comunidad. La estrategia apoya a su formación como futuros promotores culturales.

\section{DISEÑO DE LA ESTRATEGIA}

La estrategia que se ha diseñado permitirá vincular a los estudiantes con la gestión de las instituciones socioculturales mediante el desarrollo de acciones que serán puestas en práctica. Para lograr la efectividad de la estrategia, se han propuesto tres objetivos específicos:

1) Acercamiento a las instituciones socioculturales existentes en el municipio de San Cristóbal.

2) Capacitación a profesores y estudiantes de la carrera de Estudios Socioculturales para su gestión.

3) Gestión de proyectos para las instituciones socioculturales existentes en el municipio de San Cristóbal.

Cada uno de estos objetivos cuenta con un conjunto de acciones para desarrollarse, las cuales se citan en el siguiente apartado.

OBJETIVO 1: ACERCAMIENTO A LAS INSTITUCIONES SOCIOCULTURALES EXISTENTES EN EL MUNICIPIO DE SAN CRISTÓBAL.

Este objetivo va dirigido a los estudiantes de la carrera y los profesores pertenecientes a esta. Para llevarlo a cabo se plantean las siguientes acciones:

$\diamond \quad$ Organización de recorridos guiados por las instituciones con la finalidad de conocer y familiarizarse con ellas. Preparar estudiantes como guías de los propios recorridos.

$\diamond \quad$ Realización de acciones de divulgación en la intranet $y$ demás espacios adecuados, donde se expongan las características específicas de cada institución, sus principales funciones $y$ su historia en general, así como, las actividades que desarrollan.

$\diamond \quad$ Desarrollo de talleres para debatir el papel que posee el estudiante de la carrera, en la gestión de las instituciones socioculturales universitarias y en la gestión de la extensión universitaria, como parte de una labor de sensibilización y de creación de un clima de interés en la promoción de actividades culturales.

$\diamond \quad$ Organización de encuentros con fundadores y trabajadores de las instituciones socioculturales. 
$\diamond \quad$ Convocatoria a concursos asociados a la labor de estas instituciones socioculturales.

$\diamond \quad$ Edición de boletines sobre la vida cultural de las instituciones socioculturales.

$\diamond \quad$ Propuesta y organización de actividades vinculadas al objetivo de cada una de las instituciones.

Esta estrategia se dirigirá a todos los estudiantes y profesores de la carrera de Estudios Socioculturales haciendo énfasis en los estudiantes de primero $y$ segundo año, trabajando en función de lograr que los docentes ejerzan una labor de acompañamiento.

Los proyectos educativos de la brigada presentan una breve caracterización del grupo, un diagnóstico psicopedagógico, la situación política ideológica del grupo, el problema, el objeto de estudio y el objetivo fundamental del año. Además, tiene dos dimensiones, la dimensión curricular que incluye lo referente al currículo de la carrera y la dimensión extensionista que incluye todo lo referente a actividades extracurriculares.

Por su dinámica, la estrategia tendrá una intención esencialmente motivadora, de manera que genere la necesidad, el interés y los deseos de los estudiantes $y$ docentes de vincularse a la vida de las instituciones socioculturales, es decir, que los incentive a generar propuestas a partir de que interioricen el significado que tienen en su formación, en el desarrollo del sentido de pertenencia a la universidad, en el reconocimiento que alcanzarán entre los restantes miembros de la comunidad intra y extrauniversitaria $y$ en la utilidad que tiene desde su futura condición de promotores culturales.

\section{OBJETIVO 2: CAPACITACIÓN A PROFESORES Y ESTUDIANTES DE LA CARRERA DE ESTUDIOS SOCIOCULTURALES PARA SU GESTIÓN}

La capacitación propone preparar a profesores y estudiantes de la carrera de Estudios Socioculturales, con el propósito de que influyan de manera decisiva en el proceso de gestión de estas instituciones.

Dentro de las acciones encaminadas a darle cumplimiento al objetivo planteado, se han propuesto las siguientes: $\diamond \quad$ Diagnóstico de las necesidades de aprendizaje en los estudiantes $y$ profesores de la carrera de Estudios Socioculturales, para determinar las principales necesidades que presentan en cuanto a la gestión de estas instituciones.

$\diamond \quad$ Diseño de un Programa de Capacitación con la finalidad de formar a los involucrados en la gestión de las instituciones socioculturales.

$\diamond \quad$ Organización de las acciones propuestas en el Programa de Capacitación.

$\diamond \quad$ Ejecución de las acciones previstas en el Programa de Capacitación.

$\diamond \quad$ Evaluación del impacto que tendrá el Programa de Capacitación para los involucrados.

$\diamond \quad$ Selección de los facilitadores de las acciones contenidas en el Programa.

$\diamond \quad$ Preparación de la información bibliográfica en diferentes formatos para la retroalimentación.

OBJETIVO 3: GESTIÓN DE PROYECTOS PARA LAS INSTITUCIONES SOCIOCULTURALES EXISTENTES EN EL MUNICIPIO DE SAN CRISTÓBAL

La gestión de un proyecto sociocultural para los estudiantes de la carrera de Estudios Socioculturales que involucre a las instituciones socioculturales del territorio, consiste en la vinculación de los estudiantes y profesores con el desarrollo de las instituciones, utilizando al máximo el potencial de la carrera y logrando su incorporación a la vida universitaria y local.

El proyecto sociocultural debe responder a los intereses y necesidades de la carrera, el cual se desarrollará a través de equipos en los que se designará un responsable. Los equipos estarán integrados por los estudiantes y profesores de la carrera, quienes serán protagonistas íntegros de la puesta en marcha y ejecución del proyecto.

Los espacios para el desarrollo del proyecto serán el Centro Universitario Municipal de San Cristóbal, las instituciones socioculturales del Municipio, así como, otros espacios que designen los participantes.

Este planteamiento podrá materializarse a través de los proyectos educativos de la brigada y particularmente, mediante 
los proyectos sociales que se diseñan en su dimensión extensionista.

Los responsables de la ejecución y puesta en marcha del proyecto serán la Dirección de Extensión Universitaria del Centro Universitario Municipal de San Cristóbal en conjunto con la Dirección de la carrera de Estudios Socioculturales y cuyas acciones se incluirán según corresponda, en el Plan de Trabajo y entre las acciones del Proyecto Educativo de las brigadas de los años correspondientes.

El proyecto propuesto se fundamenta en la promoción de la cultura local y a la vez, en la formación sociocultural del joven universitario dentro de la Universidad, convirtiéndola en el centro cultural más importante de la comunidad. Se sustenta en un sistema de actividades a corto $y$ largo plazo que dinamicen el desarrollo sociocultural del estudiante universitario. El proyecto propone la promoción de la cultura local de San Cristóbal, a partir del desarrollo de actividades por parte del grupo de estudiantes.

Dentro de las acciones encaminadas a darle cumplimiento al objetivo planteado se proponen las siguientes:

$\diamond \quad$ Creación de espacios de debate y reflexión con los actores sociales: estudiantes y profesores de la carrera $y$ trabajadores de las instituciones socioculturales.

$\diamond \quad$ Organización del Proyecto Sociocultural.

$\diamond \quad$ Selección y puesta en marcha del Proyecto Sociocultural.

1) Creación de un espacio dedicado a la poesía con participación de poetas san cristobalenses para la promoción de su obra literaria. Este espacio durará alrededor de 2 horas y media, según los intereses de los participantes.

2) Creación de un espacio dedicado al teatro con participación de los principales representantes del teatro en San Cristóbal, con el fin de establecer un conversatorio sobre la historia del teatro en el Municipio debido a la gran tradición teatral de la zona.
3) Creación de un espacio dedicado a las artes plásticas con participación de los principales pintores del Municipio y otros, como son los alumnos de la Universidad, quienes se dedican a la pintura, asimismo se conversará sobre su obra para su promoción. Además de conocer sobre artistas que ya no se encuentran en la localidad.

4) Creación de un espacio dedicado al debate social, en el cual se conozca sobre diferentes temas de interés social como: la droga, las enfermedades de transmisión sexual, el alcoholismo y el tabaquismo.

5) Creación de un espacio dedicado a tertulias, donde se debata sobre los escritores del Municipio y cómo contribuyen con su obra al desarrollo sociocultural.

6) Creación de un espacio dedicado a los mártires de la localidad, donde se debata sobre estos y los internacionalistas del Municipio, además de invitar a sus familiares y conocer su vida. Se darán a conocer diferentes monumentos que llevan los nombres de estas personas.

\section{EJECUTANTES}

Estudiantes de la carrera, directivos de Extensión Universitaria y el coordinador de la carrera de Estudios Socioculturales.

\section{PARTICIPANTES}

Estudiantes y profesores de la carrera de Estudios Socioculturales.

\section{INSTITUCIONES PARTICIPANTES}
a. Sede Universitaria Municipal
b. Órganos Locales del Poder Popular
c. Casa de Cultura
d. Museo
e. Galería de arte
f. Biblioteca municipal 
g. Librería municipal

h. Sectorial de salud

FECHA DE EJECUCIÓN: SEPTIEMBRE 2012

\section{PLANIFICACIÓN DE LA EVALUACIÓN}

La planificación de la evaluación será responsabilidad de los que dirijan el proceso, lo que implica una preparación previa.

Se propone planificar evaluaciones sistemáticas a través de las actividades que desarrollan los estudiantes, profesores y trabajadores de las instituciones en cada una de las estrategias específicas, evaluaciones parciales al culminar cada semestre y una evaluación final, antes de culminar el curso escolar.

La planificación implica tener en cuenta el qué, cómo, cuándo y dónde evaluar:

$\diamond \quad$ Determinar los objetivos a evaluar.

$\diamond \quad$ Precisar las actividades y acciones a través de las cuales se evaluará el cumplimiento de las estrategias específicas.

$\diamond \quad$ Conocer en qué momento se realizarán los cortes parciales $y$ finales.

$\diamond \quad$ Determinar los escenarios donde serán realizadas las evaluaciones (actividades prácticas en las instituciones socioculturales y en la docencia).

\section{DETERMINACIÓN DE LOS INDICADORES DE EVALUACIÓN}

En la determinación de los indicadores de evaluación estarán implicados todos los participantes en el desarrollo de las estrategias, aunque en diferentes niveles o grados.

En tal sentido, esta labor deberá ser conducida por la dirección de la Facultad y de la Carrera, la Dirección de Extensión Universitaria $y$ representantes de las instituciones socioculturales universitarias.

Estos factores propiciarán los mecanismos de consulta que utilizarán, para garantizar la participación de los restantes actores sociales implicados en la definición y aprobación de los indicadores, a fin de lograr una adecuada concertación previa a la evaluación.
Los indicadores deberán ser precisos y atenderán los elementos esenciales que midan el cumplimiento del objetivo general de la estrategia y los objetivos específicos, teniendo en cuenta que la estrategia se materializará siempre y cuando:

$\diamond \quad$ Se familiarice a los estudiantes y profesores de la carrera de Estudios Socioculturales con las instituciones socioculturales universitarias a través de las acciones propuestas.

$\diamond \quad$ Se capaciten a los estudiantes y profesores de la Carrera $y$ a los trabajadores de las instituciones con el objetivo que se involucren en el proceso de gestión de las mismas.

$\diamond \quad$ Se pongan en práctica los proyectos socioculturales, teniendo en cuenta cada una de las necesidades e intereses de los actores implicados y las propias instituciones.

\section{INDICADORES}

1) Correspondencia de la propuesta con las necesidades de participación de los estudiantes de la carrera de Estudios Socioculturales del cum de San Cristóbal.

2) Coherencia interna de la propuesta con las posibilidades de los estudiantes de la carrera de Estudios Socioculturales del cum de San Cristóbal.

3) Rigor científico de las acciones y direcciones contenidas en la estrategia.

4) Lenguaje científico utilizado en la estrategia.

5) Las acciones son coherentes con las necesidades de los estudiantes de la carrera de Estudios Socioculturales del cum de San Cristóbal.

6) La propuesta contribuirá a insertar a los estudiantes de la Carrera en la gestión de las instituciones socioculturales existentes en el municipio de San Cristóbal.

7) Existencia de condiciones para la aplicación de la propuesta.

8) Factibilidad de aplicación de la propuesta. 


\section{EJECUCIÓN DE LA EVALUACIÓN}

La autoevaluación se realizará por los participantes en cada una de las acciones seleccionadas. Se realizará una evaluación parcial al finalizar cada semestre, sobre la base de los conocimientos adquiridos y se realizará una evaluación al finalizar el curso.

La coevaluación se realizará semestralmente $y$ al finalizar el curso se hará una evaluación, en la cual los implicados se evaluarán unos a los otros manifestando las habilidades adquiridas.

La heteroevaluación es la que realizará el personal que dirija el proceso a los miembros del grupo y se podrá realizar semestralmente, de manera que permita adoptar las medidas correctivas necesarias.

La combinación de la autoevaluación, la coevaluación y la heteroevaluación facilitará el seguimiento sistémico y la retroalimentación necesaria y apoyará el desarrollo de un adecuado ejercicio de la crítica y la autocrítica, en un clima de honestidad $y$ franqueza que favorezca la participación dinámica de todos los involucrados en el proceso.

\section{CONCLUSIONES}

El estudio del comportamiento de la participación protagónica de los estudiantes de la carrera de Estudios Socioculturales permitió determinar que aunque el surgimiento de la carrera ha constituido un cambio cualitativo $y$ cuantitativo en el desarrollo sociocultural de la Universidad, aún es insuficiente la participación de los estudiantes de dicha carrera en la gestión de las instituciones socioculturales existentes en el Municipio.

La estrategia propuesta se estructura sobre la base de tres objetivos específicos: el primero, dirigido al acercamiento a las instituciones socioculturales; el segundo, dirigida a la capacitación a profesores y estudiantes de la Carrera de las instituciones socioculturales y el tercero, estará dirigida a la gestión de proyectos para las instituciones socioculturales donde el estudiante deberá tomar parte como protagonista íntegro de cada una de las acciones que se llevarán a cabo.

\section{BIBLIOGRAFÍA}

Alarcón Ortíz, Rodolfo. "Discurso de clausura". Encuentro Binacional de Directores de Extensión y Difusión Cultural MéxicoCuba. La Habana. Cuba, 1994.

Álvarez de Zayas, Carlos M. La universidad como institución social. Sucre, Bolivia: Universidad Andina Simón Bolívar, 1996.

Ballester, Rafael. El desarrollo cultural. España: Federación Española de Universidades Populares de España, 1996.

Cabrera Ruiz, Isaac I. La extensión universitaria en la educación superior cubana: proyecciones, incidencias y gestión. Villa Clara, Cuba: Universidad Central Marta Abreu de Las Villas, 2005.

De la Riva, Fernando. "Gestión participativa de las asociaciones". Selección de Lecturas. Segunda Parte. Trabajo Comunitario. La Habana, Cuba: CIE "Graciela Bustillo", 2001.

Fadda, Giuletta. Participación como encuentro: discurso político y praxis urbana. Caracas, Venezuela: Editorial Arte Científico, 2002.

González González, Gil Ramón y González Fernández-Larrea, Mercedes. Programa Nacional de Extensión Universitaria. La Habana. Cuba, 2004.

Linares, Cecilia. "La participación". Diálogo $y$ debate en el contexto cubano. La Habana, Cuba: Centro de Investigación y Desarrollo de la Cultura Cubana Juan Marinello, 2004.

Marcovitch Raventós, E. Instrumentos de éxito para la promoción sociocultural. Quito, Ecuador: Casa de la Cultura Ecuatoriana, 2004.

Universidad de Pinar del Río. Proyecto Educativo de 4to año de la carrera de Estudios Socioculturales. Universidad de Pinar del Río.

Russeau Buenaventura, Pupo. Instrumentos teóricos y metodológicos para la gestión cultural. Colombia: Fondo Mixto de Promoción de la Cultura y las Artes del Atlántico, 1999.

Santos Gutiérrez, Sinesio. "La gestión de la extensión universitaria desde una 
perspectiva específica". Revista Cubana de Educación Superior XXI (3). CEPES. La Habana, Cuba: Universidad de la Habana, 2001.

Universalización Portal del Ministerio de Educación Superior de Cuba (MES). En: $<$ http://www.mes.edu.cu>

Universidad de Pinar del Río. Estrategia Maestra de la carrera de Estudios Socioculturales. Pinar del Río, Cuba: Centro Universitario Municipal (cum) San Cristóbal-Universidad de Pinar del Río.
Universidad de Pinar del Río. Estrategia Maestra de Extensión Universitaria. Pinar del Río, Cuba: Centro Universitario Municipal de San Cristóbal-Universidad de Pinar del Río.

Vecino Alegret, Fernando. "La universalización de las universidades: retos y perspectivas". Pedagogía. La Habana, Cuba: Palacio de las Convenciones, 2003.

Fecha de ingreso: 18/01/2012

Fecha de aprobación: 07/05/2012 
